\title{
Common features of the cerebral perforating arteries and their clinical significance
}

\author{
Vuk Djulejić $^{1}$ - Slobodan Marinković ${ }^{1} \cdot{\text { Vedrana } \text { Milić }^{2} \text { - Biljana Georgievski }}^{3}$. \\ Melanija Rašić ${ }^{1}$ Milan Aksić ${ }^{1}$ • Laslo Puškaš ${ }^{1}$
}

Received: 25 May 2015 / Accepted: 27 May 2015 / Published online: 13 June 2015

(C) Springer-Verlag Wien 2015

Dear Editor,

I would like to thank Matthieu Delion and his colleagues for their compliments regarding our article [1]. At the same time, I am sorry for having not cited their valuable paper [2] on the insular branches of the middle cerebral artery.

In general, there are some discrepancies in the terminology and definition of the insular and perforating branches. As we mentioned in our article, there are three main types of the branches of the cerebral arteries: the leptomeningeal, perforating and choroidal [3]. The superficial leptomeningeal vessels give rise to the penetrating branches, i.e. the cortical, subcortical and medullary twigs; the latter of which supply most of the white matter of the cerebral hemispheres, i.e. the centrum semiovale and the corona radiata [4]. On the other hand, the deep perforating vessels mainly originate from the basal cerebral arteries and supply the central hemispheric region, including the basal ganglia and the internal capsule.

Slobodan Marinković

mocamarinkovic@med.bg.ac.rs

Institute of Anatomy, Faculty of Medicine, University of Belgrade, Dr. Subotić 4/2, 11000 Belgrade, Serbia

2 Clinic of Children Neurology and Psychiatry, Faculty of Medicine, University of Belgrade, Belgrade, Serbia

3 Department of Radiology, Clinic "Sveti Sava", University of Belgrade, Belgrade, Serbia
Accordingly, the mentioned long insular arteries, which arise from the M2 and M3 segments of the MCA, have nothing to do with the perforating vessels of the MCA from both the morphological aspect and the region of supply. These long insular vessels simply belong to the penetrating (medullary) twigs, which were not the subject of our article.

As regards the short and medium-sized insular vessels, some of them can be classified as the "atypical" perforators. However, we presented only the typical perforating arteries, i.e. those with certain common features, as was clearly described in our paper. For that reason, we did not mention the atypical perforators, such as those to the claustrum, the cerebellar roof nuclei and the hypothalamus.

In any case, I am grateful to Delion and co-workers for their comment on our article, and for presenting the findings of their research.

Conflicts of interest None.

\section{References}

1. Djulejić V, Marinković S, Milić V, Georgievski B, Rašić M, Puškaš L (2015) Common features of the cerebral perforating arteries and their clinical significance. Acta Neurochir (Wien) 175:743-754

2. Delion M, Mercier P (2014) Microanatomical study of the insular perforating arteries. Acta Neurochir (Wien) 156:321-334

3. Marinković S (1997) General characteristics of the cerebral arteries. In: Marinković S, Gibo H, Brigante L, Milisavljević M, Donzelli R (eds) Arteries of the brain and spinal cord. Anatomic features and clinical significance. De Angelis Editore, Avellino, pp 1-10

4. Duvernoy H, Delon S, Vannson JL (1981) Cortical blood vessels of the human brain. Brain Res Bull 7:519-579 